\title{
High speed preprocessing system
}

\author{
M SANKAR KISHORE
}

Seeker Head Laboratory, Research Centre Imarat, Vignyana Kancha, Hyderabad 500069 , India

MS received 6 November 1997; revised 27 August 1999

\begin{abstract}
In systems employing tracking, the area of interest is recognized using a high resolution camera and is handed over to the low resolution receiver. The images seen by the low resolution receiver and by the operator through the high resolution camera are different in spatial resolution. In order to establish the correlation between these two images, the high-resolution camera image needs to be preprocessed and made similar to the low-resolution receiver image.

This paper discusses the implementation of a suitable preprocessing technique, emphasis being given to develop a system both in hardware and software to reduce processing time. By applying different software/hardware techniques, the execution time has been brought down from a few seconds to a few milliseconds for a typical set of conditions. The hardware is designed around i486 processors and software is developed in PL/M. The system is tested to match the images obtained by two different sensors of the same scene. The hardware and software have been evaluated with different sets of images.
\end{abstract}

Keywords. Image processing; preprocessing; hardware design.

\section{Introduction}

In systems employing tracking, the area of interest (AOI) is recognised using a highresolution camera and is handed over to the low-resolution receiver for further tracking (Kishore 1989). Acquisition and recognition of an object depend on field of view (FOV), object size, sensor resolution etc. The number of pixels an object occupies in the image plane depends on object size, range, FOV of the sensor and optics size. Because of the size limitations on the system, the object may occupy only a few pixels in the image plane. Schemes like zooming-in optics cannot be used for the same reason. For object recognition, at least 6 to 7 lines are required as per Johnson's criteria (Johnson 1958). Hence, object recognition is not possible through the low resolution sensor. Thus there is a requirement of a high-resolution sensor (HR) and a preprocessing system (PS). Ground-based HR can have bigger optics, narrow field of view, high resolution sensor etc. (Kishore 1989).

Initially, HR and low-resolution systems (LRS) are co-mounted on a platform. The object acquired through HR is supposed to appear in the FOV of the LRS. After recognizing 
the object through HR, the scene around the object can be processed to make it compatible to LRS image in spatial resolution. The processed image can be used as a reference image for locating its position in the LRS's FOV by image correlation techniques. LRS continues updating the reference information at a sufficiently fast rate and keeps tracking the area.

The sensors in the LRS and HR from which the reference is generated may have different spatial resolution. In order to match two images by correlation, the two images should have the same spatial resolution. To make the two images have the same resolution, a suitable preprocessing technique (Boland et al 1977) is applied to the high resolution image.

The suitable preprocessing algorithm has been described in a report by Boland et al (1977). The performance of the preprocessing algorithm (PA) has been studied using COMTAL-Micro Vax Image processing system and is found to be satisfactory (Kishore 1989). The automatic target handing-over system has been designed and developed using iAPX 86 family of microprocessors (Kishore 1994). This paper discusses the design implementation of preprocessing algorithm using i486 processor. It also discusses the computational complexity of the algorithm, criticality of the execution/processing time and steps taken to reduce the execution time.

\subsection{Preprocessing algorithm}

Let us consider two images of the same scene, taken by two different sensors: one with HR and the other with Low Resolution (LR). Let $W h$ be the horizontal scale factor and $W v$ be the vertical scale factor between the HR and LR images. The simplified preprocessing algorithm is as follows.

$$
\begin{aligned}
\operatorname{LR}(i, j)= & 1 /(W v \times W h)\}\left\{\sum_{m=u+1}^{x} \sum_{n=v+1}^{y} \operatorname{HR}(m, n)\right. \\
& +\sum_{n=v+1}^{y}[(u-(i-1) W v \operatorname{HR}(u, n)+(i W v-x) \operatorname{HR}(x+1, n)] \\
& +\sum_{m=u+1}^{x}[(v-(j-1) W h) \operatorname{HR}(m, v)+(j W h-y) \operatorname{HR}(m, y+1)] \\
& +(u-(i-1) W v)(v-(j-1) W h) \operatorname{HR}(u, v)+(j W h-y) \operatorname{HR}(u, y+1) \\
& +(i W v-x)(v-(j-1) W h) \operatorname{HR}(x+1, v) \\
& +(j W h-y) \operatorname{HR}(x+1, y+1)\}
\end{aligned}
$$

where,

$$
\begin{aligned}
& U=\{(I-1) W v+1\} \\
& V=\{(j-1) W h+1\} \\
& X=\{i W v\} \\
& Y=\{j W h\}
\end{aligned}
$$


The algorithm, converts the $\operatorname{HR}(i, j)$ image into an $\operatorname{LR}(i, j)$ image with $W h$ and $W v$ scale factors. The algorithm averages real number of pixels $(W h, W v)$. It is computationally complex.

\section{Methods to reduce computational complexity}

In one typical configuration, the $W h$ and $W v$ are 19.26 and 18.15 respectively. That is, to generate ONE pixel in LR, 19.26 pixels horizontally and 18.15 pixels vertically have to be considered. In other words, to generate $16 \times 16$ (say) LR image from a HR image with these scaling factors, 6890 real number (floating point) multiplications, 2351023 integer additions and 21101 floating point additions have to be performed. Thus the computation is very complex. In addition to the algorithm complexity, the computation time mainly depends on the processor speed and the application software code.

\subsection{Factors affecting execution time}

In this section, critical points affecting processing/execution time and methods to reduce execution time are discussed.

(a) Processing time can be reduced by making a dedicated hardware instead of processorbased hardware. Flexibility of changing parameters reduces.

(b) By increasing the processor speed, execution time can be reduced.

(c) By parallel processing, processing time can be further reduced. Hardware complexity increases and the dialog between the processors becomes more cumbersome than the algorithm.

(d) Since the algorithm is computationally very complex, PL/M 86 language is chosen instead of assembly language to develop the application software. The PL/M compiler converts into machine language more efficiently than any other high level language.

(e) 8086 Processor has four segments, viz. code, data, stack and extra segments. In the 8086/87 based system, HR image occupies three segments. To access each pixel/data, comparison of the pixel address to know to which segment it belongs to and accordingly jumping to that segment is necessary. That is, the processor has to execute one additional comparison and jump instruction to access each pixel. This can be overcome by using high-end processors. Alternatively the software can be made into three modules. One module computes the LR image data pertaining to the data present in one full segment. One module computes the LR image data corresponding to the data present in another full segment. The third module is to generate LR image data pertaining to that data present in the overlapping region. By this type of partition, comparison and jump instructions will be present only in the third module. Thus computation time due to these extra instructions is reduced.

(f) Since the scaling factors are fixed, the address of each pixel can be pre-computed and stored in the memory. This reduces execution time.

\subsection{Study to reduce execution time}

A study has been carried out to determine the effect of fractional pixels on boundaries for large scaling factors ( $W h$ and $W v$ ). The RMS error due to neglecting partial pixels is of the 
order of 1 in 256 gray levels (Kishore 1999). This is because the adjacent regions in an image are highly correlated. In the case of large scaling factors, by neglecting the boundary pixels, processing time can be reduced.

\subsection{Coarse re-sampling}

A study has been carried to determine the effect of coarse re-sampling. The error due to coarse re-sampling is of the order of 1 or 2 in 256 gray levels (Kishore 1999). Even when sampling rate is $4 \mathrm{MHz}$ and alternate HR lines only are considered, the RMS error is of the order of 1 or 2 gray level in 256 gray levels. Re-sampling with $W h$ and $W v$ beyond a reasonable number 4 (approximately) does not improve the accuracy considerably. Thus by coarse re-sampling, processing time can be further reduced.

\section{Hardware design}

The hardware has been designed and developed using an 80486 DX 25/33 MHz processor. To increase processing speed, internal $8 \mathrm{~K}$ cache memory is designed for optimum operation. To store intermediate values and final LR images local memory of $(128 \mathrm{k} \times 8) \times 4$ size is provided. For storing the application software $128 \mathrm{~K} \times 8 \times 4$ size EPROM space is provided. To reduce execution time (code fetching), the application code is transferred from EPROM to local memory on power ON and executed. The control logic and the chip selects are generated using EPLDs. Two serial communications links are provided using 8274 and to get the required baud rate programmable timer $82 \mathrm{C} 54$ is provided. For more details see figure 1 .

In the hardware design there are two main and critical signal paths. (a) Digitizing and writing the video data in the memory at HR rate, and (b) once the data are ready in the memory, reading the data and generating the LR image. Thus the execution time mainly depends on (i) processor speed, and (ii) the time taken for fetching video information/data

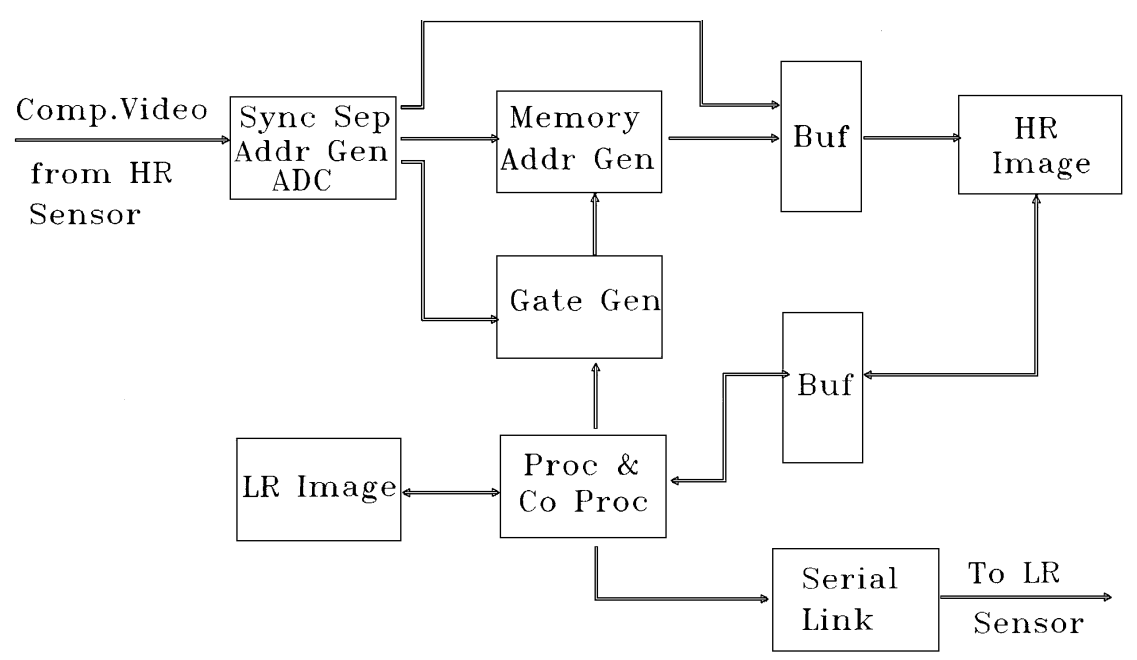

Figure 1. Hardware block diagram. 
from memory. The input image coming from the HR camera can be written in memory using high-speed logic devices in real time. The CPU accesses the data and generates the LR image. That is, the CPU should have access to read and write the memory. Thus to avoid two address and data bus clashes they are multiplexed.

Composite video signals coming from the HR camera consist of Horizontal Sync (HS), Vertical Sync (VS) and Video Sync (HS, VS) signals are separated using the sync separator. The video is sampled at HR sensor clock rate and 8-bit digital data are obtained through a fast A/D converter. For getting pixel and line addresses, FCT series digital counters are used and are initialized by clock, HS and VS signals. Components like counters and buffers used between the A/D converter and the memory are of ACT/FCT series to match the input data rate.

The processor receives the airborne vehicle co-ordinates from the HR sensor and generates the left, right, top and bottom edges of a gate around the target. The pixel address, left and right edges are compared to get the $\mathrm{X}$ gate. Similarly, line address, bottom edge and top edges are compared to get the $\mathrm{Y}$ gate. Using $\mathrm{X}$ and $\mathrm{Y}$ gates, an $\mathrm{XY}$ gate signal is generated. This signal is used for enabling the memory so that only the target image area is stored.

Separate sets of counters are used for pixel address and line address for addressing the memory. The pixel counters are cleared with gated HS and X gate signal. Similarly, the line counters are cleared with gated VS and Y gate. These address lines go to memory for addressing different locations. When the processor wants to read the memory it sends the address to the memory and reads the data. To avoid the conflict between these two address and data buses, all the address, data lines and control lines are connected through multiplex /buffers as shown in figure 1.

\section{System software}

The present application software is developed in PL/M language. The application program mainly consists of two parts viz. (a) Built-in self-test, and (b) application. These options can be selected by means of a switch provided externally.

As explained in the previous sections, to reduce execution time, the addresses of the pixels are pre-computed and stored as lookup tables. Since the scaling factors are fixed, the preprocessing algorithm is partitioned such that the comparison and JUMP statements are minimized by which fetching time is reduced drastically.

On power ON, the USARTs (serial communication interface) and programmable timer/ counter are initialized. The USARTs are initialized in async mode, with one stop bit, no parity, eight-bit character length, $16 x$ baud rate and enabled transmit \& receive (DTS \& RTS). Programmable timer counters 0 and 1 are initialized to get square waves of 9600 baud frequency, required for USART for transmission and reception.

After initializing all the peripherals in the above mode, the processor waits for the start signal. It checks the HR sensor serial link. Once the serial link test is completed, HR sensor sends the target coordinates ( $\mathrm{X} \& \mathrm{Y}$ ) and checksum to the system. Then the processor computes the left, right, bottom and top edges of the target and applies the predefined boundary conditions.

The computed target edges are outputted to the comparators through the ports with synchronization with the field pulse VS. For synchronization, the processor checks the VS by reading through an input port bit. Here both high and low levels of the pulse are taken 
into consideration for checking. This avoids false synchronization. A predefined size of the image around the target is captured in the memory. The processor computes the LR image data by applying the preprocessing algorithm. The computed low-resolution data are transmitted to the LRS through a serial link.

\subsection{Built in test facility}

The self-test can be initiated by putting the external switch and pressing the reset. The serial link provided for connecting the low resolution sensor may be connected to a PC or computer terminal. If a PC is connected then it is sensed by the S/W, CPU computes the low-resolution data and sends them to a PC. The data can be displayed on a PC to see the input/output picture quality. In the case of a computer terminal, the CPU checks the status of each part and displays corresponding messages on a terminal. To monitor the status in the absence of a PC/terminal, a 7-segment display is provided.

It checks the sync signals and gate generation. In gate generation, the gate signals have to be monitored on DSO. The memory is tested by writing and reading fixed values, incremental and random patterns. The corresponding status is displayed on a terminal. Facility to display the captured image as per the selected gate size is also provided.

\section{Test results}

To evaluate the system in the laboratory, both in hardware and software, a high-resolution visible camera and LR camera are co-mounted on a movable platform. An existing correlation tracker is used as a low-resolution sensor (LRS). The image is taken from the HR sensor and processed using the present system. The generated and LRS images are transmitted to a PC through a serial link and correlation is performed using the software. The

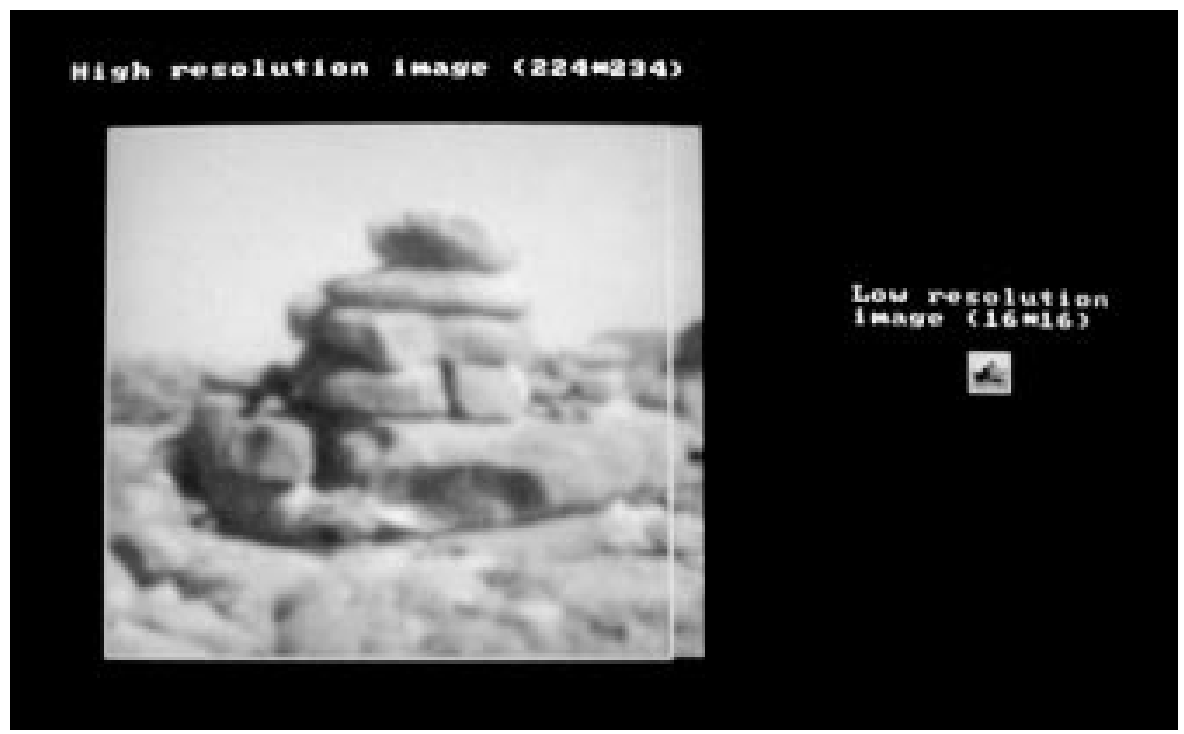

Figure 2. Sample 1: High resolution image $(224 \times 234)$ and converted low resolution image $(16 \times 16)$ 


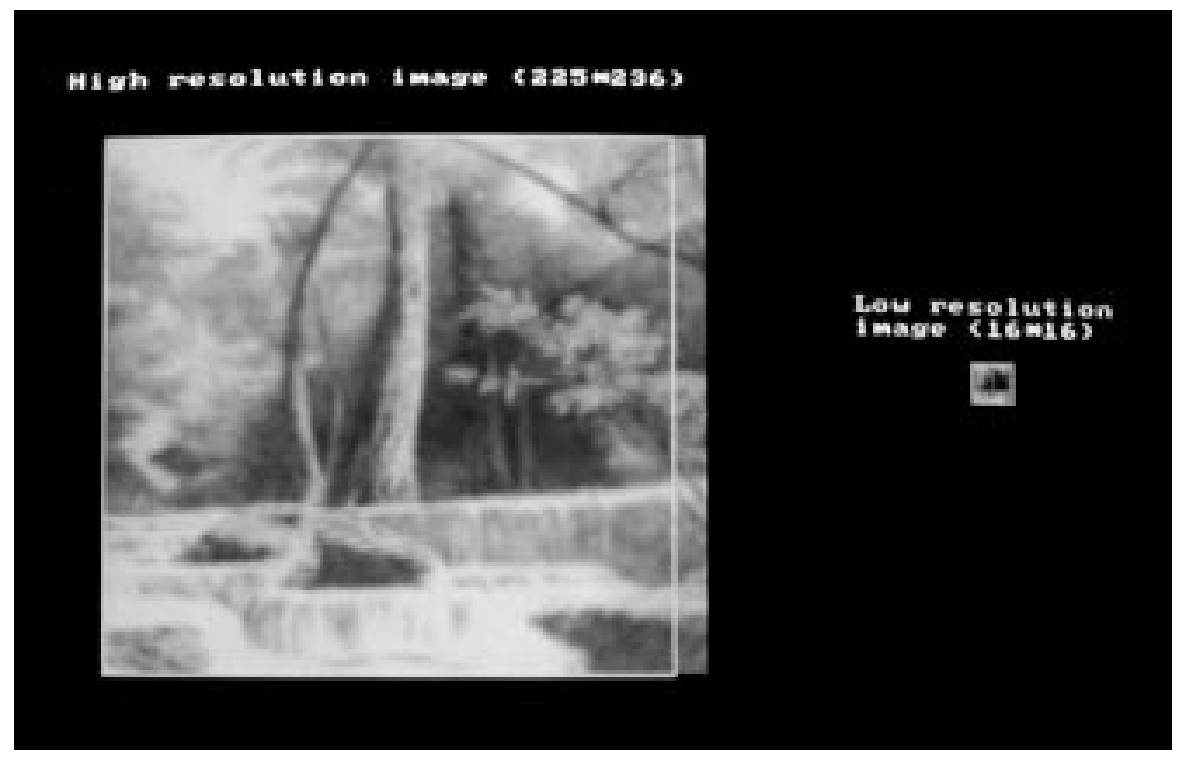

Figure 3. Sample 2: High resolution image $(225 \times 236)$ and converted low resolution image.

generated low-resolution image is also transmitted to the LRS, which is properly locked to the area of interest on its search image. This is repeated several times to validate the system. The hardware used came in three PCBs of standard double Euro card of size of $233.4 \mathrm{~mm} \times 220 \mathrm{~mm}$ excluding the motherboard.

The system has passed all MIL-STD qualification tests as below:

* Low temperature test: $\quad-30^{\circ} \mathrm{C}$ for duration $16 \mathrm{~h}$ and last one hour operational.

$*$ High temperature test: $\quad+55^{\circ} \mathrm{C}$ for duration $16 \mathrm{~h}$ and last $10 \mathrm{~h}$ operational.

* Damp heat test: $\quad+45^{\circ} \mathrm{C}$ and $95 \% \mathrm{Rh}$ for duration $16 \mathrm{~h}$ and last one hour operational.

* Vibration: $\quad$ As per MIL STD 810 E.

*EMI/EMC: $\quad$ CS06, CE03, RS 03 and RS 02.

For illustration, two sample photographs (figures 2 and 3) are presented. The white line on the high-resolution image shows the size of the image considered for generating the low-resolution image. The execution time in each case has been computed with the present 486 processor-based system and the old 8086/87 based system (Kishore 1994). The timings are tabulated in table 1 . The timings meet the project specifications very satisfactorily.

Table 1. Results. Comparison of execution times between two systems.

\begin{tabular}{llcc}
\hline Scaling factors & Samples & $8086 / 76$ system & 80486 system \\
\hline$W v=15.32$ & Image I & $7.9 \mathrm{~s}$ & $222 \mathrm{~ms}$ \\
$W h=14.19$ & Image II & $1.36 \mathrm{~s}$ & $52.18 \mathrm{~ms}$ \\
$W v=6.34$ & & & \\
$W h=8.15$ & & & \\
\hline
\end{tabular}




\section{Conclusions}

A preprocessing system has been designed with i486 processor based hardware with highspeed logic devices. The techniques/methods discussed in the paper have been implemented in the new design, both in hardware and software. Due to the on-chip cache and other hardware logic, speed has increased 5.6 times and due to the software modularity, the speed has increased 4.3 times. That is, $5 \mathrm{~s}$ time (say) has become $900 \mathrm{~ms}$ due to hardware and $200 \mathrm{~ms}$ due to software. All the control logic has been fused in EPLDs and firmware in $E^{2}$ PROMS to maintain the security of the design. The software is designed in modular format with elaborate built-in test facility.

I wish to express my sincere gratitude to Mr K V S S Prasad Rao for encouragement. My deep sense of gratitude is due to Dr K Veerabhadra Rao, Mr T V Rao, and Mr N R Iyer, DRDL for their kind advice and guidance provided throughout this work. I am also thankful to Dr S B Gadgil, Mr B V Rao and Mr K Rambabu for their useful suggestions at various stages and to all colleagues, who helped directly and indirectly to complete this work.

\section{References}

Boland J S, Pinson L J, Kane G R, Honnell M A, Peters E G 1977 Automatic target handoff using correlation techniques. Technical report, Auburn University, Alabama USA, pp 57-63

Johnson J 1958 Analysis of image systems. Proceedings of Image Intensifier Symposium, US Army Engineering Research Development Laboratory, Fort Belvoir, pp 100-110

Kishore M S 1989 Image preprocessing system. Proceedings of International Technical symposium on Optic \& Optical Electronics, Applied Science and Engineering, San Diego. SPIE vol. 1153, pp $140-145$

Kishore M S 1994 Automatic Target Handing over system. Defense Sci. J. 44: 69-80

Kishore M S 1999 Target handing over system using pyramid processing. Defense Sci. J. 49: 41-48 